\title{
2d Frictional B-Spline Smoothed Mortar Contact Problems Part I: Matching Phase
}

\section{Kallel $\mathrm{A}^{1 *}$ and Bouabdallah $\mathrm{S}^{2}$}

${ }^{1}$ Pôle Universitaire Léonard de Vinci, De Vinci Reserach Center, Paris La Défense, France

${ }^{2}$ Laboratoire Roberval UMR 7337, Université Technologique de Compiègne, 60205 Compiègne, France

\begin{abstract}
Simple averaged normal vector, Hermit polynomial, Cubic B-Spline curve and other technique are used to smooth surface for frictional contact problem between deformable bodies in context of large deformation. Mortar approach is combined with augmented Lagrange formulation to treat the contact constraints. A spline interpolation is also employed for the linearization of the kinematic contact constraints. Cubic B-Spline is applied at the boundary of contact element while maintaining a classical Lagrange interpolation at the interior. The quality of the contact pressure obtained with B-Spline is better than that achieved with a hall Lagrange discretization. The performance of the proposed framework is illustrated with representative two dimensional numerical examples.
\end{abstract}

\section{Keywords: Matching phase; Mortar}

\section{Introduction}

Usually for accurate contact problems one used to include finite element discretization applying the standard NTS (Node-ToSegment) formulation [1] in the transmission of contact constraints. Using NTS method involve loosing accuracy of displacements and stresses in the contact area due to the fully nodal-wise contact constraints. To circumvent NTS purview, mortar method is initially used for unilateral small deformation contact problem [2]. It has been successfully extended to solve large deformation contact problems [3]. Mortar method is a discretization technique initially used in domain decomposition for non-matching grids. It is applied by connecting two different discretized surfaces using Lagrange multipliers. Then it is used to treat continuously contact problems $[4,5]$. The transmission of the contact constraints is performed by a weak projection of the displacement from one contacting body to the next using integrals defined in $C^{1}$ contact surface.

On the other hand, the contact strength calculations also depend on accurate and smoothed interactions that impact global as the local contact pressure and the tangential tractions description. Mortar approach is a method of contact interaction treatment through an exact evaluation of surface integrals contributing to the weak formulation. It will be combined with discreet satisfaction of contact constraints [6-13].

The unsmoothed finite elements $\left(\mathrm{C}^{0}\right.$ continuity generally) are the most widely used discretization techniques for the calculation of contact problems. It has long been recognized that the unsmoothed discretization leads to convergence problems. It could result also high oscillation in the tangential effort for the frictional contact problems [14-19]. To overcome these issues, various techniques have been developed as Hermit, Bezier, B-Spline [20-23]. The mortar approach allows smooth contact constraints through generated in the weak formulation of the problem. In the literature, there are also some smoothing techniques applied directly on the contact surface [24-26].

Cubic B-Spline $[27,28]$ is used to describe the contact surface and replace the $\mathrm{C}^{0}$ continuous Lagrange polynomials usually indented in classical finite element method. Linear Lagrange interpolation used in classical finite element method is insufficient to describe a curved surface unless the use of a finer element meshes which increase the problem size. The normal vector is not defined continuously which causes errors in the imposition of the kinematic contact conditions, and in the accuracy of the gap calculation as shown in Figure 1.

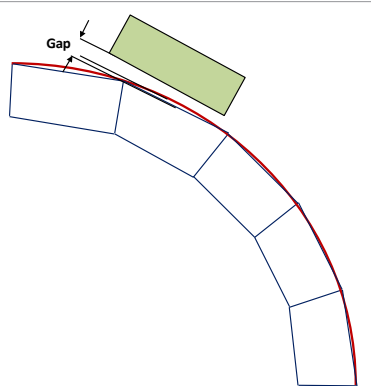

Figure 1: Contact surface and normal vector based on standard finite element interpolation.

The smoothing procedure operates only on the contact surface. An exact characterization of the initial geometry (or very close to) helps to obtain a smooth description which can then be combined with mortar approach.

\section{Mechanical Model}

Now we present a study of a two-dimensional large deformation contact problem with different formulations. Figure 2 illustrates two $2 \mathrm{D}$ bodies $\beta_{1}$ and $\beta_{2}$ occupying their initial configuration $\Omega_{0}^{(2)}$ and $\Omega_{0}^{(2)}$ of $\mathbb{R}^{2}$ then $\Omega^{(1)}$ and $\Omega^{(2)}$ in the current configuration. $\mathbb{R}^{2}$ have a direct orthogonal reference $\left(0, \vec{e}_{1}, \vec{e}_{2}\right)$. Note $\Gamma^{1}$ and $\Gamma^{2}$ are the borders of $\Omega_{0}^{(2)}$ and $\Omega^{(2)}$ in the reference configuration then $\gamma^{1}$ and $\gamma^{2}$ are the borders of $\Omega_{t}^{(1 \rho}$ and $\Omega_{t}^{(2)}$ in the current configuration.

At the initial configuration, a given point $X^{i} \in \Omega_{0}^{i}$ maps to $x^{i}$ at the current configuration by:

*Corresponding author: Kallel A, Pôle Universitaire Léonard de Vinci, De Vinci Reserach Center, Paris La Défense, France, Tel : +33 1411670 00; E-mail : achraf.kallel@devinci.fr

Received June 14, 2017; Accepted June 30, 2017; Published July 04, 2017

Citation: Kallel A, Bouabdallah S (2017) 2d Frictional B-Spline Smoothed Mortar Contact Problems Part I: Matching Phase. J Appl Mech Eng 6: 272. doi: 10.4172/2168-9873.1000272

Copyright: (c) 2017 Kallel A, et al. This is an open-access article distributed under the terms of the Creative Commons Attribution License, which permits unrestricted use, distribution, and reproduction in any medium, provided the original author and source are credited. 


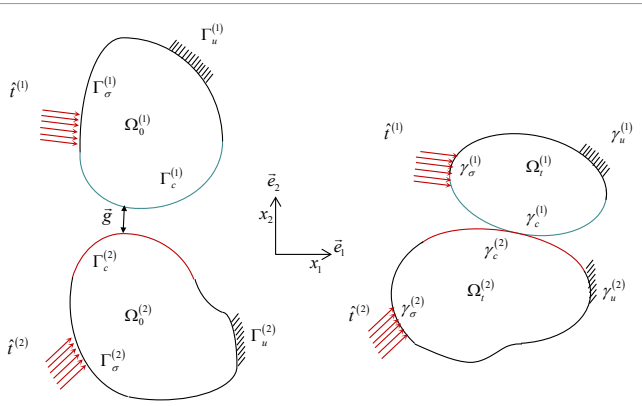

Figure 2: 2D contact problem and notations.

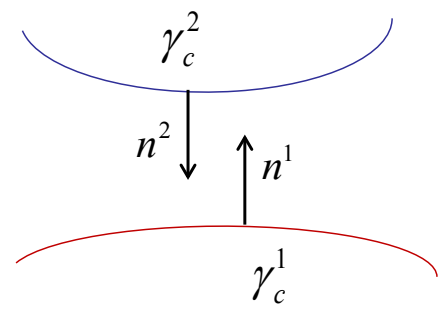

Figure 3: Normal vectors in the contact interface.

$$
x^{i}=x^{i}\left(X^{i}, t\right) \quad \text { with } \quad x^{i}: \Omega_{t}^{i} \times[0, T] \rightarrow \square^{2}
$$

The boundary of each body is split into three separate parts, such as:

$$
\begin{aligned}
& \Gamma_{u}^{(i)}+\Gamma_{\sigma}^{(i)}+\Gamma_{c}^{(i)}=\Gamma^{(i)} \\
& \Gamma_{u}^{(i)} \cap \Gamma_{\sigma}^{(i)}=\Gamma_{\sigma}^{(i)} \cap \Gamma_{c}^{(i)}=\Gamma_{u}^{(i)} \cap \Gamma_{c}^{(i)}=\varnothing
\end{aligned}
$$

These three parts are defined as follows:

$\Gamma_{u}^{(i)}$ is the part of $\Gamma^{(i)}$ in which displacements $\hat{u}^{i}$ are imposed.

$\hat{t}^{(i)}$ is the part in which surface forces $\hat{t}^{(i)}$ are imposed.

$\Gamma_{u}^{(i)}$ is the part in contact with another body.

$\Gamma_{u}^{(i)}, \Gamma_{\sigma}^{(i)}$ and $\gamma_{u}^{i}$ are transformed on $\gamma_{u}^{i}, \gamma_{c}^{i}$ and $\gamma_{c}^{i}$ in the current configuration.

The resolution of a normal contact problem (frictionless) returns to the determination of the interaction between a point $x^{1}$ on the contact boundary $\gamma_{c}^{1}$ and the closest point $x^{2}$ of $\gamma_{c}^{2}$. This interaction results in a normal force of resistance to penetration of the two bodies, it is noted $t_{N}$. The distance between these two points is commonly called normal gap and recorded $g_{N^{*}}$. In a resolution of the problem by the finite element method, there are three approaches to the nature of points $x^{1}$ and $x^{2}$ :

- All pairs of points $x^{1}$ and $x^{2}$ are nodes meshes: it is the node to node approach (denoted in the following NTN).

- One of the two points corresponds to a mesh node: this approach is said node to segment (noted in the NTS continued).

- If the two points are any points of contact interface, it is in the case of a segment to segment approach (denoted in the following STS).

Note by 'd' the distance between a master point $x^{1}$ given on the surface $\gamma_{c}^{2}$ and a point $x^{2}$ on the slave surface $\gamma_{c}^{2}$. This function is expressed by:

$$
d=\left\|x^{1}-x^{2}\right\|
$$

The relationship that connects a point of the master surface $X^{1}$ in the reference configuration to its counterpart $x^{1}$ in the present configuration after the application of a displacement $u^{1}$ is given by:

$x^{1}\left(X^{1}, t\right)=X^{1}+u^{1}\left(X^{1}, t\right)$

Similarly, for a point on the slave surface, the relation can be written:

$x^{2}\left(X^{2}, t\right)=X^{2}+u^{2}\left(X^{2}, t\right)$

$n^{1}$ is the outgoing normal vector of the surface $\gamma_{c}^{1}$ at the point $x^{1}$ and $n^{2}$ the normal vector outgoing of the surface $\gamma_{c}^{2}$ (Figure 3).

The minimum distance between a point on the slave surface $x^{2}$ and its closest counterpart belonging to the master surface $x^{-1}$ is given by:

$$
g_{N}=\left(x^{2}-\bar{x}^{1}\right) \cdot \bar{n}^{1}
$$

The gap must always be positive or zero to apply the nonpenetration between the two bodies in contact.

$$
g_{N=}\left(x^{2}-x^{-1}\right) \cdot \mathrm{n}^{-1} \geq 0
$$

The normal force representing a contact pressure is conventionally negative when the gap is zero. It vanishes when the gap becomes positive and the two bodies are no longer in contact. There is a complementary relationship between the normal force $t_{N}$ and the gap $g_{N}$. These three relationships can be written as conditions Karush-Kuhn-Tucker:

$$
g_{N} \geq 0, t_{N} \leq 0, g_{N} t_{N=} 0
$$

The relations in (1.8) are usually used for solving optimization problems. Traditionally, in mechanical contact problem such relations are called the conditions of Hertz-Signorini-Moreau. For a contact problem with friction [14-17] applying these conditions leads to a geometric expression as inequality:

$$
\int_{\Omega}[\delta E: S] d \Omega_{0} \geq \int_{\Omega} \rho_{0} b . \delta u d \Omega_{0}+\int_{\Gamma_{\sigma}} \hat{t} . \delta u d \Gamma_{\sigma}
$$

We treat the contact problem in the static approach by establishing the balance of contact interactions in a well-defined contact field $\gamma_{c}$. We calculate the reduced stresses in the reference configuration of a point of the current configuration by the first stress tensor Piola-Kirchhoff. The equilibrium equation is formulated in the reference configuration. By combining the balance with the displacement boundary conditions imposed on $\Gamma_{\sigma}^{i}$ and surface forces imposed on $\Gamma_{\sigma}^{i}$, we can define the following problem:

$$
\begin{aligned}
& \operatorname{Div}(F . S)^{(i)}+\rho_{0}^{(i)} b^{(i)}=0 \text { on } \Omega_{0}^{(i)} \\
& u^{(i)}=\hat{u}^{(i)} \quad \text { on } \Gamma_{u}^{(i)} \\
& t^{(i)}=\hat{t}^{(i)} \quad \text { on } \Gamma_{\sigma}^{(i)}
\end{aligned}
$$

With $F$ : Gradient transformation

$S$ : Second Piola-Kirchhoff stress tensor

$P_{0}:$ Density in the reference configuration

$b$ : Volume Force

$t$ : Surface Force

The second stress tensor Piola-Kirchhoff is obtained by deformation of Green-Lagrange $E^{(i)}=\frac{1}{2}\left(F^{T} F-\mathrm{I}\right)^{(i)}$ using the Kirchhoff St. Venant material behaviors:

$$
S^{(i)}=C^{(i)}: E^{(i)}
$$

With $C^{(i)}$ is the constitutive tensor. 
The existence and uniqueness of the solution for a contact problem without friction in small displacement is shown in [17]. The work of the contact forces $G_{C}\left(u, \delta u, t_{c}\right)$ contributes to the work of the internal forces $G_{\text {ext }}^{(i)}(\delta u)$ and the external forces $G_{e x t}^{(i)}(\delta u)$ to give the following global work expression where $t_{c}$ represents the contact force.

$$
G\left(u, \delta u, t_{c}\right)=\sum_{i=1}^{2}\left(G_{\text {int }}^{(i)}(u, \delta u)+G_{e x t}^{(i)}(\delta u)\right)+G_{C}\left(u, \delta u, t_{c}\right)=0
$$

Which

$$
\begin{aligned}
& G_{\text {int }}^{(i)}(u, \delta u)=\sum_{i=1}^{2} \int_{\Omega_{0}^{(i)}}\left[\delta E^{(i)}: S^{(i)}\right] d \Omega_{0}^{(i)} \\
& G_{e x t}^{(i)}(\delta u)=\sum_{i=1}^{2}\left\{\rho_{0}^{(i)} \int_{\Omega_{0}^{(i)}} b^{(i)} \cdot \delta u^{(i)} d \Omega_{0}^{(i)}-\int_{\Gamma_{\sigma}} \hat{t}^{(i)} \cdot \delta u^{(i)} d \Gamma_{\sigma}^{(i)}\right\} \\
& G_{C}\left(u, \delta u, t_{c}\right)=\sum_{i=1}^{2} \int_{\Gamma_{c}^{(i)}} t_{c}^{(i)} \cdot \delta u^{(i)} d \Gamma_{c}^{(i)}
\end{aligned}
$$

where $u$ are the displacements and $u$ their variations.

The virtual work arising from contact constraints is computed at the slave surface $\Gamma_{c}^{(1)}$. The expression of $G_{c}$ depends on the choice of used formulation. There are different techniques in the literature to force the contact conditions [29,30]. For the frictional contact problem, the sliding conditions lead to the definition of a tangential component in the contact interface $t_{t}$.

In practice only a part of the candidate contact surfaces is active. This portion of the surface is the zone in which the normal gap is zero. Cancelling it will trigger the calculation of the contact force and the new positions of the bodies in contact. The rest of the interface is presented as an inactive contact region that can become active. The determination of the status of the active contact areas is performed by using an algorithm known as the Active Set Strategy whose application is detailed in [24,31-34]. We suppose that we know at this stage active area of the contact, and then we can apply the contact constraints to the problem.

\section{Discretization of the Contact Interface}

The first calculation of an analytical solution of a contact problem was made by Hertz in [34] between a sphere and an infinite half space. Johnson [35] extended the analytical solution with solids having other geometries. The analytical resolution of a contact problem is limited to simple geometries that can be described analytically. In order to treat a contact problem between complex geometry bodies, an approximation of the solution using the finite element method was developed [17, 3638]. In these early attempts to modelling contact problems between deformable body by the finite element method, applications were limited to the case of small deformations and small displacements hence the use of linear theories. In this case, the mesh sizes used in the body interface were compliant. The discretization proposed by Francavilla [39] prohibits any slipping between the two bodies in contact. The first application of this method to a contact problem with large displacement is developed by Hughes [40]. The application of the contact conditions is bye the node to node method detailed by Kikuchi and Oden [17]. The node to node method (NTN) may be considered as the simplest numerical method for treating the contact.

The mathematical resolution of the NTN method is to just adding forces to the node that compensate the contact force. Its implementation requires an algorithm that handles the activation or deactivation of the nodal forces of contact. The discretization mesh of two bodies in contact must be homogenous for this method to can use it. This method is then restricted to conforming meshes for a contact problem in small deformation and small relative movements in the interface.

The normal gap $g_{N}$ between the two bodies in contact (Figure 4) is calculated by projection. The gap always has a positive value to avoid the overlap of the two bodies (condition of non-penetration). In general case, the nodes are distributed in an arbitrary manner on the contact interface view that the two bodies are not necessarily mesh with the same mesh sizes (Figure 5). And if that is the case, this approach is limited to cases of small deformation in the elastic range.

A segment node approach (NTS) was developed by Simo [41] to discretize the improper meshing contact interface (Figure 5). In the NTS method, the conditions of contact and friction are applied between: on the one hand a node of the contact surface of the first body and on the other side a segment belonging to the other body. Initially, this method was applied to the two-dimensional contact problems with elastic constitutive law in large displacement. An extension to the case of nonlinear constitutive laws like the hyper-elastic model and elasticplastic law was then presented [18,42-45]. This discretization technique did not permit the satisfaction of patch-test [46]. The NTS method has a difficulty to well transmit the contact forces from one body to another for a perfectly flat interface as shown in [47].

The patch test is presented by a rectangular block of 4 UL (length unit) lengths and 2 UL in width support on its lower edge. Another rectangular block, which the dimension is 2 UL by $1 \mathrm{UL}$, is based on the first, as shown in Figure 6. The two blocks are subjected to a uniform pressure of $1 \mathrm{~F} / \mathrm{UL}^{2}$ on their upper surfaces. A neo-Hooke behaviors is considered for the two blocks which the Young modulus is $E=1000$ $F / U L^{2}$ and Poisson coefficient correspond to $v=0.3$. The lower block is discretized with $16 \times 8$ elements when the top block is discretized with $20 \mathrm{x} 4$ elements. The analytical solution of this problem has been discussed in [3], the value of the expected vertical stress is constant over all of the mesh elements is $\sigma_{y y}=-1 F / U L^{2}$.

\section{Material characteristics:}

\section{$E=1000 \mathrm{~F} / U L^{2}$}

$v=0.3$

Loading:

$p_{N=} 1 F / U L^{2}$

Analytical solution:

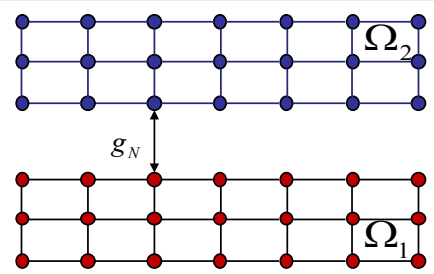

Figure 4: Contact interface between two homogeneous meshes: NTN discretization.

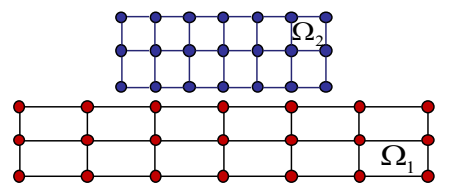

Figure 5: Contact interfaces between two non-conforming meshes. 


$$
\sigma_{\text {vertical }=}-1 \mathrm{~F} / U L^{2}
$$

The NTS method remains the most used in a large range of industrial computer codes. In the literature, there are algorithms for the NTS approach using double pass [48] for solving the problem of the patch test. This means that at each problem increment is solved twice: on the first stage, one affects the choice of master and slave surfaces and on the second stage masters and slave's surfaces are exchanged. This technique is limited to the case of linear interpolation elements and presents convergence problems for higher order elements for which the compatibility conditions said (Inf-Sup) are not satisfied. To reduce these problems of convergence without excessively penalizing the computation time, we find in the literature using the local refinement of the mesh in the contact area [49]. A detailed report on the patch test with the NTS discretization can be found in [50], where a new approach combining linear and quadratic shape functions is also available.

In the NTS method, we also need to define a master surface and a slave surface. The choice of the slave surface among the two surfaces in the interface is theoretically arbitrary. In practice, the slave surface is associated with the finer mesh or the least rigid body. The minimum distance (commonly known normal gap) which separates the two

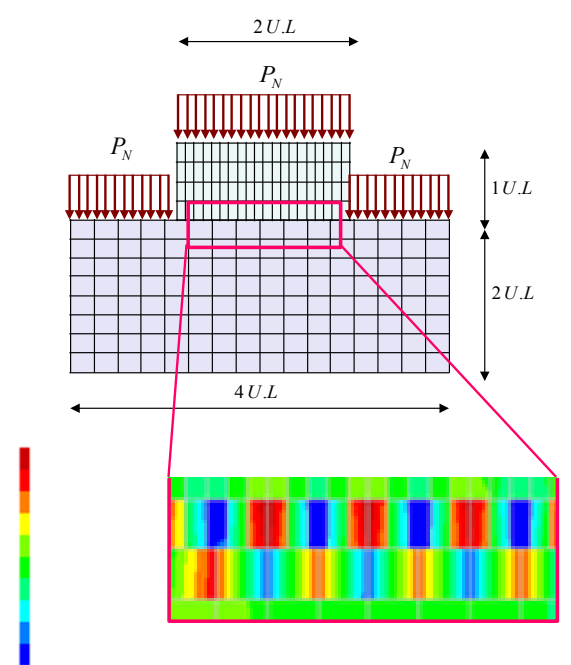

Figure 6: Patch-test contact problem.

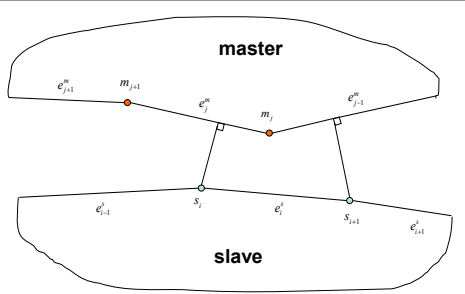

Figure 7: Matching and projection in the NTS method.

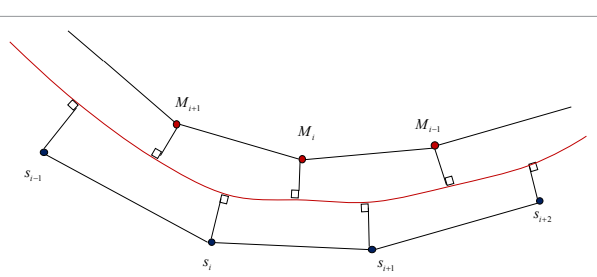

Figure 8: Discretization of the contact interface using contact segments [66].

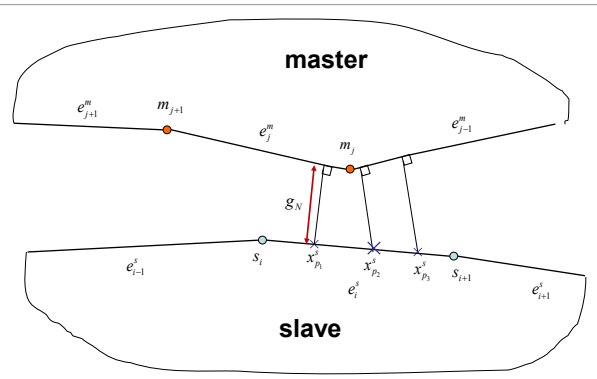

Figure 9: Matching and projection for mortar method (3 integration point for each slave segment).

bodies in contact is calculated by orthogonal projection of all the nodes of the slave surface on the master surface (Figure 7). The conditions of non-penetration for the NTS approach is as the incapacity of the slave node to enter the master segment associated after projection.

The segment to segment method (STS) been proposed first by Simo for the case of two-dimensional contact problem. This first approach comes down to the use of an intermediate contact surface that occurs naturally from the discretization of the contact interface in contact segments (Figure 8). Recently this discretization has been generalized effectively to contact problems in three dimensions. This technique of discretization was coupled with the approach of mortar elements inspired by the domain decomposition method [50,51]. Discretizing with mortar approach, part of the STS method family, is stable and passes the patch test but its implementation is difficult and requires a lot of technical expertise. This technique has been successfully applied for normal contact problems $[3,8,9]$ and for contact problems with friction $[2,11,13,53,54-57]$.

The mortar method is initially a technique used for solving decomposition problems of non-conforming domains [57,58]. It has been adapted to modeling unilateral contact problem without friction or between different bodies [4,59]. This technique is well suited to the application of the contact conditions in a discrete system consisting of a non-conforming mesh in the interface. It is based on the use of Lagrange multipliers representing the contact force resulting nodes [7]. An intermediate surface, as a reference surface for the Lagrange multipliers, is exploited for the first uses of this technique [2]. In this paper, the use of this method is generalized to be applied for solving a contact problem with friction in large displacement and sliding. The bearing surface of the Lagrange multipliers can be one of the two contact surfaces (master or slave) [5,60]. A modified dual method for the case of contact between geometrically complex surfaces is detailed by Flemiscli [61] wherein the two contact surfaces succeed to be a reference surface for the Lagrange multipliers. An algorithm close to NTS double-pass method is deployed. A generalization of this technique was developed by Popp A [62] to a 3D contact problem.

Contact and friction constrains are applied in a weak way and the discretization should check Brezzi-Babuska conditions [63-65]. The algorithmic method and its theoretical expression are detailed in $[24,30,66]$. Non-penetration conditions will be applied on the integration points of the slave's segments and not on the slave nodes as for the NTS approach (Figure 9).

In the literature, smoothing techniques were always applied in a local way. The idea in this work is to combine the local smoothing of the geometry description with an overall smoothing of the contact constraints application. The mortar approach is an accurate assessment of the integrals in the interface to meet the contact constraints. A 
contact problem could be over-conditioned if it is combined with other constraints such as thermo-mechanical problem $[67,68]$ or the dynamic contact problem [11]. In this case, the use of the mortar method proves very important to solve a contact problem because it can smooth different constraints (contact, friction thermal ...) in the interface under an integral form $[7,9,10]$. If we add to the mortar method for the contact constraints enforcement a direct smoothing technique applied in the interface, as in $[17,24,25]$, we can guarantee a superior robustness in the resolution algorithm. An 'exact' characterization (or very close) to the geometry of the initial analysis helps in the obtaining of a smooth description which can be then combined with the mortar approach for the resolution of a contact problem in finite deformation.

\section{Mortar Approach Description}

The mortar approach will be the discretization method of the contact interface adopted for this work. In the contact interface, the master surface is designated ${ }^{1}$ and the slave surface as $\Gamma^{2}$. In the mortar approach, we use the terms mortar surface $\Gamma^{m}$ (master) and non-mortar surface $\Gamma^{n m}$ (slave).

The mortar method allows coupling subdomains from the contact surfaces of the two body defined by two independent meshes. In this approach, the term 'segments' means the elements of the discretized contact surfaces (Figure 10). The discretized mortar surface (same of the non-mortar) is the union of all the segments that comprise it:

$$
\Gamma^{n m} \approx \bigcup_{j=1}^{N} \Gamma_{j}^{n m} \quad \Gamma^{m} \approx \bigcup_{i=1}^{M} \Gamma_{i}^{m}
$$

Exhibitor's 'nm' and ' $\mathrm{m}$ ' indicate respectively non-mortar and mortar. The contact interface has respectively $(\mathrm{M})$ and $(\mathrm{N})$ and nonmortar and mortar segments. Since the meshes of two bodies in contact are not conforming, the two surfaces $\Gamma^{n m}$ and $\Gamma^{m}$ are not identical. For the rest, the non-mortar surface will serve as integration support for the calculation of different expressions $\left(\Gamma_{c}=\Gamma^{n m}\right)$, variables $\xi$ and $\zeta$ designate respectively the natural coordinated in the mortar and the nonmortar segment. With the finite element method, the displacement is discretized through shape functions. One was limited in this study to the use of a linear interpolation of the displacement field on the border. The shape functions are given by:

$$
\begin{array}{ll}
N_{1}^{n m}(\zeta)=1-\zeta & N_{2}^{n m}(\zeta)=\zeta \\
N_{2}^{m}(\xi)=\xi & N_{2}^{m}(\xi)=\xi
\end{array}
$$

The displacement $u$ and the position of a point of the contact interface $X$ may be written:

$$
\begin{aligned}
& \text { On a mortar segment } \Gamma^{m}: u^{m}(\zeta)=\sum_{i=1}^{2} N_{i}^{m}(\zeta) u_{i}^{m} \\
& X^{m}(\zeta)=\sum_{i=1}^{2} N_{i}^{m}(\zeta) X_{i}^{m}
\end{aligned}
$$$$
\text { On a non-mortar segment } \Gamma^{n m}: u^{n m}(\zeta)=\sum_{i=1}^{2} N_{i}^{n m}(\zeta) u_{i}^{n m}
$$
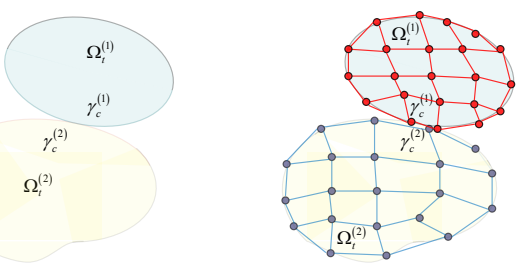

Figure 10: Discretization of the contact problem by the finite element method.

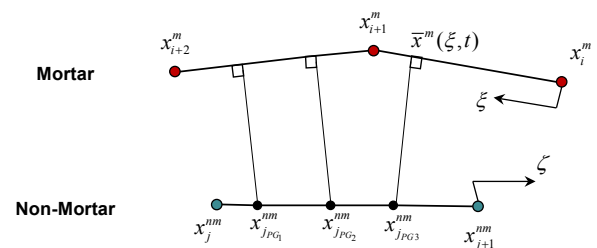

Figure 11: Projection of integration points of the non-mortar segment on mortar segments.

$$
X^{n m}(\zeta)=\sum_{i=1}^{2} N_{i}^{n m}(\zeta) X_{i}^{n m}
$$

The contribution of the contact forces to the global virtual work can be expressed as a function of different variables, depending on the formulation: The normal gap $g_{N}$, the tangent gap $g_{T}$, normal Lagrange multiplier $\lambda_{N}$, tangent Lagrange multiplier $\lambda_{T}$ and respectively their variations $\delta g_{N}, \delta \lambda_{N}, \delta \lambda_{N}$ and $\delta \lambda_{T}$, and as well as the normal contact pressure $p_{N}$. This function is the sum of $c_{N}$, contribution of normal forces of contact, and $c_{T}$ for the tangential forces.

$$
G_{c}=\int_{\Gamma_{c}} c_{N}\left(\lambda_{N}, g_{N}, \delta \lambda_{N}, \delta g_{N}\right) d \Gamma+\int_{\Gamma_{c}} c_{T}\left(\lambda_{T}, g_{T}, \delta \lambda_{T}, \delta g_{T}, p_{N}\right) d \Gamma
$$

Regardless of the choice of the formulation, the contact problem requires solving a system of nonlinear equations. After linearization of expressions $c_{N}$ and $c_{T}$ to establish the residual vector and the tangent matrix, we use the Newton-Raphson algorithm to solve the final problem. Other nonlinearities (material and/or geometric) may be added to the nonlinearity of the contact. Detail in the following the expressions of the tangent matrixes and residual vectors for different formulations. By replacing the contact surface by its discretization (2.1) we can write:

$$
\begin{aligned}
G_{c} & =\int_{\Gamma_{c}} c_{N}\left(\lambda_{N}, g_{N}, \delta \lambda_{N}, \delta g_{N}\right) d \Gamma+\int_{\Gamma_{c}} c_{T}\left(\lambda_{T}, g_{T}, \delta \lambda_{T}, \delta g_{T}, p_{N}\right) d \Gamma \\
& =\bigcup_{s=1}^{N}\left[\int_{\Gamma_{c}^{s}} c_{N}\left(\lambda_{N}, g_{N}, \delta \lambda_{N}, \delta g_{N}\right) d \Gamma+\int_{\Gamma_{c}^{s}} c_{T}\left(\lambda_{T}, g_{T}, \delta \lambda_{T}, \delta g_{T}, p_{N}\right) d \Gamma\right]
\end{aligned}
$$

In this expression we find two terms to detail: $c_{N}$ and $c_{T}$. In the case of a contact problem without friction, only the first term has a meaning. The next section is dedicated to the quantification of that term in various formulations. An extension to the case of contact with friction will be detailed in the next section.

To solve a contact problem, we have to define a relationship between the two surfaces of the contact interface $\Gamma^{n m}$ and $\Gamma^{m}$. This relationship allows associating any non-mortar point the closest mortar point. This relationship requires solving a minimization problem of the distance between the two points. We note $x^{m}\left(\mathrm{X}^{m} \mathrm{t}\right)$ and $x^{n m}\left(X^{n m} \mathrm{t}\right)$ the positions of the mortar and non-mortar points in the current configuration.

We introduced a function, noted $\mathrm{d}$, that describes the distance between a slave point (non-mortar) $x^{n m}$ given on the surface $\gamma^{n m}$ and a point $x^{m}=x^{-m}(\xi, t)$ on the mortar surface with designating $\xi$ the natural coordinate on the mortar surface (Figure 11). This relationship is given by:

$$
d=\left\|x^{n m}-x^{m}(\xi, t)\right\|
$$

The relationship that connects a point on the mortar surface $X^{m}$ in the reference configuration to its counterpart in the current configuration after the application of a displacement $u^{m}$ is given by:

$X^{m}\left(X^{m}, t\right)=X^{m}+u^{m}\left(X^{m}, t\right)$

It is the same for a point of non-mortar surface: 


$$
x^{n m}\left(X^{n m}, t\right)=X^{n m}+u^{n m}\left(X^{n m}, t\right)
$$

The distance between the two points $x^{n m}$ and $x^{-m}$ should be minimal, this condition is ensured by verifying the following equation:

$$
\left.\frac{d}{d \xi} d(\xi, t)\right|_{\xi=\xi}=0
$$

$\bar{\xi}$ is the natural coordinate value on the mortar segment which checks (2.9). The line through the point $x^{n m}$ and its projection $x^{-m}$ gives us the direction of the normal vector of the mortar segment noted $n^{m}$ and used for projection (Figure 12). The unit normal vector can be therefore defined by:

$$
a^{m}=\frac{d}{d \xi} x^{m}
$$

The tangent vector to the mortar surface on the point with the coordinate $\xi$ is:

$$
a^{m}=\frac{d}{d \xi} x^{m}
$$

The value of the natural coordinate of the point is calculated from the orthogonality relation between normal and tangent vectors:

$$
n^{m} \cdot \mathrm{a}^{-m=0}
$$

The minimum distance in the normal direction (normal gap) between the two bodies in contact is given by the expression:

$$
g_{N}=\left(x^{n m-x} x^{-m}\right) \cdot n^{-m}
$$

The gap is updated during the movement of the two bodies and its value always indicates the minimum distance between a non-mortar point $x^{n m}$ and its mortar counterpart $x^{-m}$. If the value is zero, the two bodies are in contact. If this value becomes negative then the condition of non-penetration is violated (Figure 13).

By canceling, the gap $g_{N}$ triggers the resolution of the contract. For a frictionless contact problem, a contact pressure $t_{N}$ will be exerted at the interface of two bodies. The gap and pressure are the unknowns of the problem.

The resulting contact force $\bar{t}_{c}$ on the surface $\Gamma$ has two components: a component normal to the contact surface and noted $\bar{t}_{c_{t}}$ and another tangent to this surface and noted $\bar{t}_{c_{t}}$.

$$
\overline{t_{c}}=\overline{t_{c_{n}}}+\overline{t_{c_{t}}}
$$

The bar above the force vectors indicates that it is in the current configuration. To resolve the problem, we have to write the equation balance and the contact constraints in a weak form.

The contribution of the contact forces will be calculated on each non-mortar integration point. The amount found will be reduced to non-mortar nodes. All contributions calculated on the non-mortar nodes will be used to establish the elementary matrix.

The balance of forces on the contact interface $\gamma_{c}$ gives:

$$
\bar{t}_{c}^{n m} \cdot d \Gamma^{n m}=-\bar{t}_{c}^{m} \cdot d \Gamma^{m}
$$

The virtual work of the contact forces is described by:

$$
G_{C}\left(u, \delta u, t_{c}\right)=\sum_{i=1}^{2} \int_{\Gamma_{c}^{(i)}} t_{c}^{(i)} \cdot \delta u^{(i)} d \Gamma_{c}^{(i)}
$$

Using equality (2.16), contact virtual work on the slave contact surface is written:

$$
G_{C}=-\int_{\Gamma^{m m}} \bar{t}_{c}^{n m} \cdot\left[\delta u^{n m}(X)-\delta u^{m}(\bar{X})\right] d \Gamma^{n m}
$$

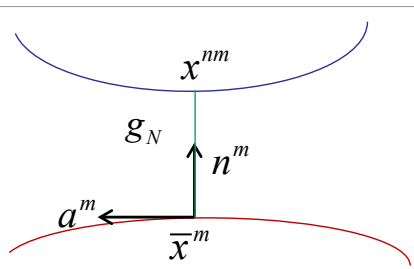

Figure 12: Tangent vector and normal vector for a mortar point.

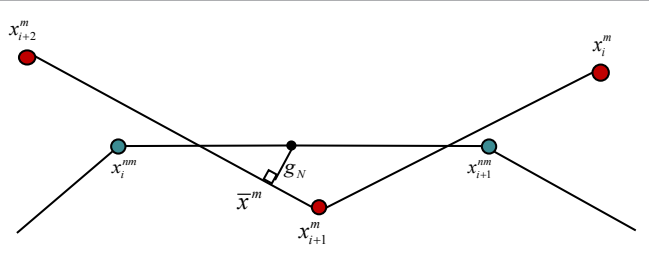

Figure 13: Non-checking of the interpenetration conditions.

For frictionless contact, (2.5) will be reduces to the normal components and is written:

$$
G_{c}=\int_{\Gamma^{n m}} \lambda_{N} \cdot \delta g_{N} d \Gamma^{n m}
$$

\section{Formulation of a Normal Contact Problem}

Solving the problem by the Newton-Raphson method requires the linearization of $c_{N}$ with respect to both contact variables $\lambda_{N}$ and $g_{N}$ then their variations $\delta \lambda_{N}$ and $\delta g_{N}$.

$$
G_{c}=\int_{\Gamma_{c}} c_{N}\left(\lambda_{N}, g_{N}, \delta \lambda_{N}, \delta g_{N}\right) d \Gamma=\bigcup_{s=1}^{N}\left[\int_{\Gamma_{c}^{s}} c_{N}\left(\lambda_{N}, g_{N}, \delta \lambda_{N}, \delta g_{N}\right) d \Gamma\right]
$$

The linearization of the function $c_{N}$ can be written as follows:

$$
\Delta c_{N}=\frac{\partial c_{N}}{\partial \lambda_{N}} \Delta \lambda_{N}+\frac{\partial c_{N}}{\partial g_{N}} \Delta g_{N}+\frac{\partial c_{N}}{\partial \delta g_{N}} \Delta \delta g_{N}+\frac{\partial c_{N}}{\partial \delta \lambda_{N}} \Delta \delta \lambda_{N}+
$$

\section{Formulation of a Contact Problem with Friction}

The contribution of the contact efforts to the global virtual work is given in (2.4) and by using the description of the contact interface in (2.5). To solve a frictional contact problem (Figure 14), the expressions of $c_{N}$ and of $c_{T}$ are to linearize according to the variables of the chosen contact formulation $[55,56]$.

The expression of the contribution of the normal contact $c_{N}$ was detailed in the previous section. It remains now to give the expression of $c_{T}$.

The expression of the virtual work of in the contact can generally be written as follows:

$$
G_{c_{s}}=\int_{\Gamma_{c}} \mathbf{t}_{c} \delta \mathbf{g} d \Gamma
$$

Knowing that the contact effort $t$ is the sum of two vectors of normal and tangent efforts as follows:

$$
\mathbf{t}_{c}=\mathbf{t}_{c_{N}}+\mathbf{t}_{c_{T}}
$$

And

$$
g=g_{N} n^{m}+\mathrm{g}_{\mathrm{T}} a^{m}
$$

The vector $\mathbf{g}$ has a normal component (2.23) and another one tangential (2.24) as shown in the Figure 2.

With $g_{N}=$ g. $n^{m}$ 


$$
\text { And } g_{T}=\mathbf{g} \cdot \frac{a^{m}}{\left\|a^{m}\right\|}
$$

For frictional contact problem (Figure 15), we can have two possible situations: stick contact or slip contact. To distinguish these two statuses, we use the law of Coulomb for the friction and we evaluate the function $f_{s}$ defined by:

$$
f_{s}=\| \mathbf{t}_{c_{T}}|-\mu| t_{c_{N}} \mid
$$

Knowing that $\mathbf{t}_{c_{T}}$ is the normal contact effort and is the Coulomb friction coefficient, it remains to determine the effort vector of tangent contact $\mathbf{t}_{c_{T}}$ to evaluate the function (2.25). $\mathbf{t}_{c_{T}}$ Is obtained by the projection of the global contact vector $t_{c}$ on the tangent plan:

$$
\mathbf{t}_{c_{T}}=\frac{1}{\left\|a^{m}\right\|^{2}}\left(\left\{a^{m}\right\} \otimes\left\{a^{m}\right\}\right) \mathbf{t}_{c}
$$

The sliding function (2.25) can be now calculated and the status of the tangent contact will be defined according to the sign of this function as follows:

$$
f_{s}\left\{\begin{array}{l}
|| \mathbf{t}_{c_{T}}|-\mu| t_{c_{N}} \mid \geq 0 \quad \text { slip } \\
|| \mathbf{t}_{c_{T}}|-\mu| t_{c_{N}} \mid<0 \quad \text { stick }
\end{array}\right.
$$

The presence of the friction adds variables to the contact problem
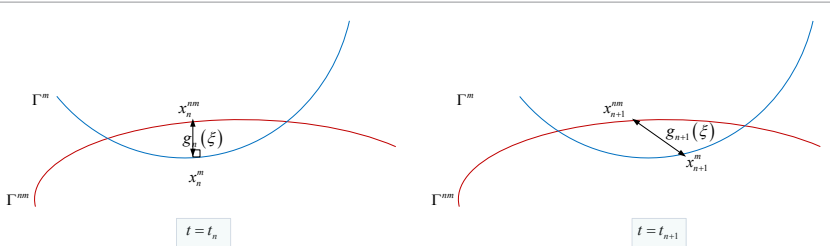

Figure 14: Frictional contact of two bodies between two load-steps.

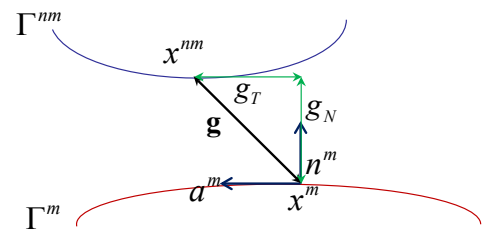

Figure 15: Calculation of the gap for a frictional contact problem.

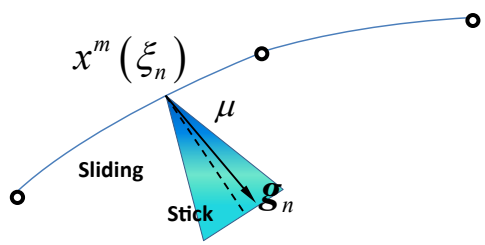

Figure 16: Position of the friction cone for a stick contact.

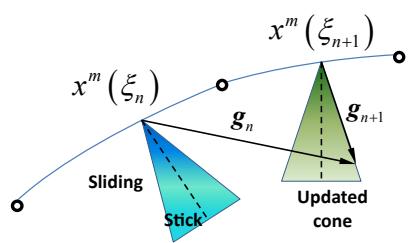

Figure 17: Updating of the friction cone for sliding contact. but also the numerical instabilities to the resolution. This instability arises from the passage of a contact point in the interface from a stick status to a sliding one or the opposite. This passage results a numerical instability which requires a specific treatment to reduce its influence on the convergence quality of the calculation. One adopt the Moving Friction Cone MFC) applied with the law of Coulomb to solve a contact problem with friction as for the two dimensional geometry by Opara [69] and for three dimensional problem in [19]. When the tangential forces overtake a certain limit, both surfaces in contact will not be any more attached but we notice a relative displacement between them. This relative displacement represents the sliding which is described by the Coulomb law as follows:

$$
\mathbf{t}_{c_{T}}=\mu\left|t_{c_{N}}\right| \frac{g_{T, t}}{\left\|g_{T, t}\right\|} \text { si } \quad\left\|\mathbf{t}_{c_{T}}\right\|>\mu\left|t_{c_{N}}\right|
$$

$g_{T, t}$ is the speed of sliding is. The coefficient of Coulomb $\mu$ is maintained constant during the contact between both surfaces. To estimate the status of an integration point on a non-mortar segment already in contact with a mortar segment, it is necessary to calculate the sliding function (2.25) with the natural coordinate $\xi$ of the point. In the case of a stick contact, the amplitude of the tangent effort $\mu\left|t_{c_{y}}\right|$ is lower on the limit of the normal effort $\mu \mid t_{c_{N}}$ evaluated by the Coulomb law. By applying the friction cone approach, every non-mortar integration point is inside a cone as shown in the (Figure 16).

If integration point in the non-mortar segment between the time step $t_{n}$ and $t_{n+1}$ shows a relative displacement in the tangential direction to the associated mortar segment (on which is made the projection for the load step $t_{n}$, the position $\xi_{n}$ is going to change another one $\xi_{n+1}$. In the case of a frictionless contact, the update of the position $\xi_{n}$ is directly made, but for the frictional contact it is necessary to verify some conditions to change its value.

If the contact effort obtained from the observed displacement is inside the friction cone, the tangent effort is lower to the limit $\mu\left|t_{c_{N}}\right|$ , the tangent displacement is not sufficient to bring out the nonmortar integration point of the cone, thus there will be no updating of the projection position $\xi_{n}$ and the vector $g_{n+1}^{s t}$ remains invariant with $\xi_{n+1}=\xi_{n}$. If the function $f_{s}$ are positive, we can conclude that the tangential effort $t_{T}$ is upper to the normal adhesion $\mu\left|t_{c_{N}}\right|$. The vector $g_{n+1}^{s t}$ will be updated and the natural coordinate after projection will be updated and will take then the new value $\xi_{n+1}$ (Figure 17).

The expression of the virtual work of the contact (2.20) depends on the choice of the formulation, we shall detail, in what follows, this expression by using first the penalty method.

\section{Smoothing Techniques}

Most of the discretization techniques of the finite element method used for the resolution of a contact problem is of continuity $\mathrm{C}^{0}$. A not smoothed discretization often leads to convergence problems either to highly oscillating contact interactions in particular for the tangential effort for a frictional contact problem. To limit some of these unwanted observations, different smoothing techniques of the contact interface for a two-dimensional problem were developed.

Among these techniques, we can quote the Hermite polynomials as in [22], [25] and Foley, [70], interpolation of cubic Bernstein in the shape of Bezier curve by Pietrzak [71] and [21] either cubic Splines as by Al-Dojayli [72] and [23]. In these last two works, splines are used locally which returns finally to the use of the Bézier curves. For the smoothing of the contact areas in the case of a $3 \mathrm{D}$ problem we find in the literature the use of Gregory's patches as in [53]. All the 
quoted works used the NTS approach for the application of the contact constraints.

In the literature, the term smoothing was often directly connected to the description of the geometry, and in particular to the surface normal vector. Indeed, the smoothing can be global for writing with a high order of continuity, as he can be local by acting on the terms of contact interactions as the normal pressure and the tangential effort.

For a contact problem, the smoothing is only applied to the contact zone to avoid any overload of calculation. By deleting the jumps of normal led by the polygonal description of the skin, the smoothing of the interface allows to improve the estimation of the contact efforts. For of large deformation problems, in spite of the smoothing of the geometry, some oscillation can persist because of the strong interactions between discretized surfaces [73].

In resolution phase of a contact problem, we notice the influence of the formulation chosen on the quality of the results and on the speed of convergence. Although the use of the augmented Lagrange method allows to stabilize the calculation and to obtain coherent results, she remains sometimes insufficient especially in the case of large deformation problems. Two neighboring not collinear segments after deformation, present a discontinuity of the tangent vector. The expression of the tangent matrix $K_{T}$ and the tangent effort $t_{T}$ for a frictional contact depends on the mortar surface tangent vector $a^{m}$. A discontinuity of this last pulled directly a problem in the calculation of these two terms. The normal vector of the mortar surface $n^{m}$ is directly connected to the tangent vector:

$$
n^{m}=\frac{\vec{e}_{3} \times a^{m}}{\left\|\vec{e}_{3} \times a^{m}\right\|}
$$

A discontinuity problem in the tangent vector leads automatically a problem of the same order for the normal vector. The normal vector of the mortar surface is used for the calculation of the gap. A discontinuity of this vector wills results problems for the existence and the uniqueness of the projection, (Figure 18). The proposed solution to this problem is to add a smoothing method to the mortar approach. The smoothing is proposed with various techniques.

\section{Method of Averaged Normal}

This method, widely used in the literature, is implemented under various variants in numerical codes. In certain works, such as [33], we calculate the average of the normal vector for every node from both normal vectors of the neighboring segments as follows:

$$
\tilde{n}_{i}^{m}=\frac{\left(n_{i}^{m}+n_{i+1}^{m}\right)}{\left\|n_{i}^{m}+n_{i+1}^{m}\right\|}
$$

The configuration of the averaged normal vector is as given in the following Figure 19:

In the paper [54], the smoothed normal vector is averaged from the normal vectors of both neighboring segments weighted by the size of these two last ones as the following formula shows:

$$
\tilde{n}_{i}^{m}=0.5 \frac{\left(l_{i+1}^{m} n_{i}^{m}+l_{i}^{m} n_{i+1}^{m}\right)}{\left\|l_{i+1}^{m} n_{i}^{m}+l_{i}^{m} n_{i+1}^{m}\right\|}
$$

The application of this method is detailed in the following (Figure 20):

The used technique in this work is the one employee in [11]. We calculate the averaged tangent vector by:

$$
\tilde{a}_{i}^{m}=0.5 \frac{\left(a_{i}^{m}+a_{i+1}^{m}\right)}{\left\|a_{i}^{m}+a_{i+1}^{m}\right\|}
$$

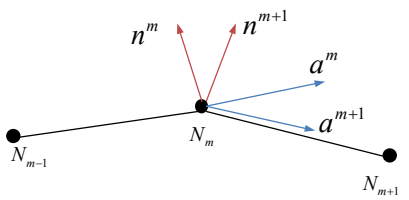

Figure 18: Normal multiple on node.

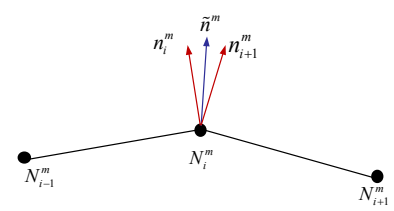

Figure 19: Averaged normal vector.

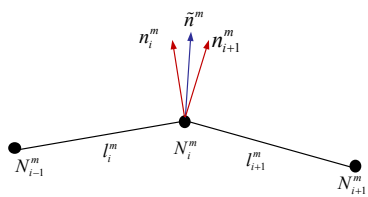

Figure 20: Averaged normal vector balanced with the elements length.

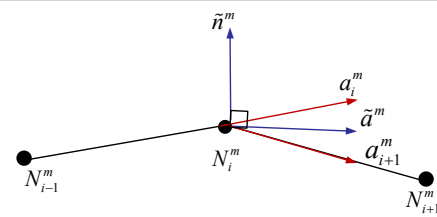

Figure 21: Averaged normal vector from averaged tangent vector.

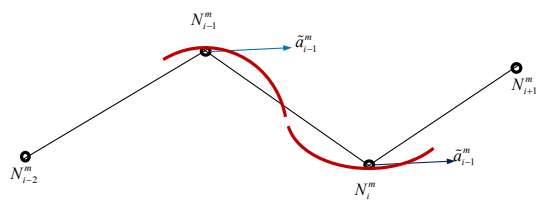

Figure 22: Generation of a geometry offset with the mesh.

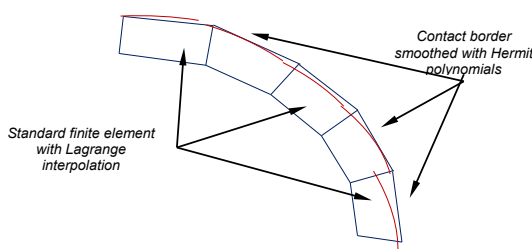

Figure 23: Elements of borders smoothed with Hermit polynomials.

Then we apply the relation (3.1) to obtain the averaged normal vector (Figure 21):

In the resolution of the contact problem, the calculation of the normal gap $g_{N}$ will be according to the averaged normal vector $\tilde{n}^{m}$ and respectively the tangent gap $g_{T}$ for a frictional problem will be calculated using the averaged tangent vector $\tilde{a}^{m}$.

To conclude, this technique avoids the jump in the normal and tangent direction near nodes. The evolution of normal in the segment remains linear and the contact surface maintains a $C^{0}$ continuity order. For a projection close to node (the value of the natural coordinate $\hat{n}^{m}$ 
very close to 0 or 1 ), we notice an improvement on the error of the gap calculation compared with a projection without averaged vector. If the direction of the averaged vector is distant from that of the original vector, a geometry offset of the meshing will be built (Figure 22). Material will be added or subtracted from the body on which these normal are defined. It can slightly perturb the mass conservation of the system. This phenomenon restricts the application of this technique for the problems in large deformation.

\section{Hermit Cubic Function}

In this section, we propose a smoothing of continuity $\mathrm{C} 1$ in the contact interface of a two-dimensional mortar problem. Let us consider linear elements with a Lagrange interpolation for the geometry discretization of bodies in contact and a description by Hermit polynomials for the border (Figure 23).

The cubic Hermit polynomials are only used for the description of the contact area [74]. The calculation of the minimal gap which separates both bodies will be assured by a projection using a new smoothed normal vector noted $\hat{n}^{m}$. The latter is normal to mortar the surface, for that reason we limited the smoothing at only this surface only (non-mortar surface is not smoothed) (Figure 24). To find the expression of the normal vector, it is necessary to calculate at first the smoothed tangent vector:

$$
\hat{a}^{m}(\xi)=\frac{\partial \hat{x}^{m}(\xi)}{\partial \xi}
$$

then we apply the relation (3.1). It remains to find a detailed expression of a mortar point $\hat{x}^{m}$ on the new curve defined by the Hermit polynomials according to its natural coordinate $\xi_{\varepsilon}[0,1]$ This expression is given by:

$$
\hat{x}^{m}(\xi)=H_{1}^{m}(\xi) x_{m-1}^{m}+H_{3}^{m}(\xi) x_{m}^{m}+H_{2}^{m}(\xi) x_{m-1, \xi}^{m}+H_{4}^{m}(\xi) x_{m, \xi}^{m}
$$

With $H_{i}^{m} \quad i=\{1,2, \ldots, 4\}$ are the Hermit polynomials. They are defined as follows:

$$
\begin{aligned}
& H_{2}^{m}(\xi)=\xi-2 \xi^{2}+\xi^{3}, \\
& H_{2}^{m}(\xi)=\xi-2 \xi^{2}+\xi^{3}, \\
& H_{3}^{m}(\xi)=3 \xi^{2}-2 \xi^{3}, \\
& H_{4}^{m}(\xi)=-\xi^{2}+\xi^{3}
\end{aligned}
$$

By applying (3.5), the equation (3.7) can be rewritten as follows:

$$
\hat{x}^{m}(\xi)=H_{1}^{m}(\xi) x_{m-1}^{m}+H_{3}^{m}(\xi) x_{m}^{m}+H_{2}^{m}(\xi) \tilde{a}_{m-1}^{m}+H_{4}^{m}(\xi) \tilde{a}_{m}^{m}
$$

With the technique of the averaged vector detailed in the previous section, we manage to smooth the pressure of contact in the neighborhood of nodes but the evolution of normal vector remains linear inside the segment. In the expression (3.8), we used the averaged tangent vectors $\tilde{a}^{m}$ obtained in (3.4).

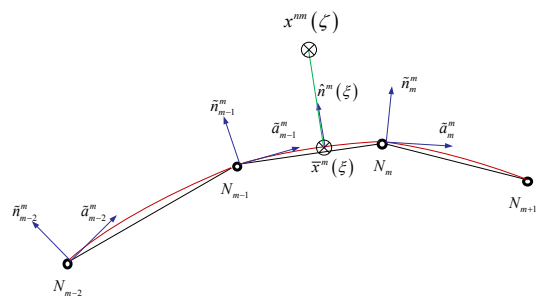

Figure 24: Projection on a smoothed curve with cubic Hermit polynomials.

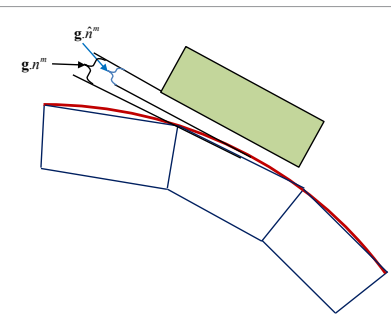

Figure 25: Influence of the smoothed normal vector on the precision of the gap calculation.

In the contact interface, an integration point of the non-mortar surface $x^{n m}(\zeta)$ is associated with its counterpart in the mortar surface $x^{-m}(\xi)$ by a projection. The vector of the gap

$$
g=x^{n m}-x^{m}(\xi)
$$

must be collinear to the smoothed normal vector $\hat{n}^{m}$ (Figure 25). It is necessary to look for the value of the natural coordinate $\xi$ which verifies this condition:

$$
\overrightarrow{\bar{x}^{m}(\xi) x^{n m}} \wedge \hat{n}^{m}(\xi)=\overrightarrow{0}
$$

By applying the expression (3.8) in (3.5), the smoothed tangent vector $\hat{a}^{m}$ can be rewritten as follows:

$$
\hat{a}^{m}(\xi)=H_{1, \xi}^{m} x_{m-1}^{m}+H_{3, \xi}^{m} x_{m}^{m}+H_{2, \xi}^{m} \tilde{a}_{m-1}^{m}+H_{4, \xi}^{m} \tilde{a}_{m}^{m}
$$

By applying (3.11) in (3.1), we can obtain the expression of the smoothed normal vector $\hat{n}^{m}$ as follows:

$$
\hat{n}^{m}=\left\{\begin{array}{l}
\hat{n}_{X}^{m} \\
\hat{n}_{Y}^{m} \\
0
\end{array}\right\}=\left\{\begin{array}{c}
-H_{1, \xi}^{m} x_{m-1_{Y}}^{m}-H_{2, \xi}^{m} x_{m_{Y}}^{m+1}-H_{3, \xi}^{m} a_{m-1_{Y}}^{m}-H_{4, \xi}^{m} a_{m_{Y}}^{m} \\
H_{1, \xi}^{m} x_{m-1_{X}}^{m}+H_{3, \xi}^{m} x_{m_{X}}^{m+1}+H_{2, \xi}^{m} a_{m-1_{X}}^{m}+H_{4, \xi}^{m} a_{m_{X}}^{m} \\
0
\end{array}\right\}
$$

By using the expression of the gap (3.9), the condition of projection (3.10) can be rewritten:

$$
\left(x_{X}^{n m}-x_{X}^{m}\right) \hat{n}_{Y}^{m}-\left(x_{Y}^{n m}-x_{Y}^{m}\right) \hat{n}_{X}^{m}=0
$$
follows:

$$
\begin{aligned}
& \left(x_{X}^{n m}-\left[H_{1}^{m}(\xi) x_{m-1_{X}}^{m}+H_{3}^{m}(\xi) x_{m_{X}}^{m}+H_{2}^{m}(\xi) a_{m-1_{X}}^{m}+H_{4}^{m}(\xi) a_{m_{X}}^{m}\right]\right) \\
& {\left[H_{1, \xi}^{m} x_{m-1_{X}}^{m}+H_{3, \xi}^{m} x_{m_{X}}^{m+1}+H_{2, \xi}^{m} a_{m-1_{X}}^{m}+H_{4, \xi}^{m} a_{m_{X}}^{m}\right]} \\
& -\left(x_{Y}^{n m}-\left[H_{1}^{m}(\xi) x_{m-1_{Y}}^{m}+H_{3}^{m}(\xi) x_{m_{Y}}^{m}+H_{2}^{m}(\xi) a_{m-1_{Y}}^{m}+H_{4}^{m}(\xi) a_{m_{Y}}^{m}\right]\right) \\
& {\left[-H_{1, \xi}^{m} x_{m-1_{Y}}^{m}-H_{3, \xi}^{m} x_{m_{Y}}^{m+1}-H_{2, \xi}^{m} a_{m-1_{Y}}^{m}-H_{4, \xi}^{m} a_{m_{Y}}^{m}\right]=0}
\end{aligned}
$$

The position of an integration point on the mortar surface $x^{m}(\xi)$ is a cubic polynomial according to $\xi$. The normal vector, and by using the expressions (3.1) and (3.5), it will be defined by a quadratic polynomial. The projection in (3.14) can be then described by a polynomial of order five as follows:

$$
\mathrm{P}(\xi)=\alpha_{1} \xi^{5}+\alpha_{2} \xi^{4}+\alpha_{3} \xi^{3}+\alpha_{4} \xi^{2}+\alpha_{5} \xi^{5}+\alpha_{6}
$$

After the determination of the expressions of the coefficients $\alpha$, Newton's iterations will be essential for the calculation of $\xi$.

In [22] and [ 25], we find that the use of the Hermit polynomials for the smoothing of the contact areas improves considerably the convergence and the robustness of the solution of a contact problem treated with NTS method. This improvement is less remarkable with the method mortar in which the contact constraints are applied in a low way and the contact efforts are transmitted on all the contact area and not point by point. The used integral in the mortar method 
produces a certain regularity of the contact pressure. That's why the low contribution on the regularity by the smoothing with Hermit polynomial compared to the NTS method.

The use of the Hermit polynomials for the smoothing of the mortar surface allows reducing the error on the calculation of the minimal gap (Figure 26) and consequently the global error. The reduction of the error on the gap was the subject of research for several works seen its big influence on the solution of the system. We find in the literature as in [9], [33] and [37] the use of quadratic elements with the mortar approach to decrease the error in the calculation of the gap.

In our case, and with linear elements, the smoothing of the contact area with the Hermite polynomials improves the description of the contact interface. The function which describes the evolution on this surface is cubic only inside segments. Thus the mortar surface will be defined as a continuation of cubic curves. Although this technique gives to the surface a continuity of order $\mathrm{C}^{1}$, its resolution algorithm is penalized by Newton's additional iterations necessary for the projection.

\section{Cubic B-Spline}

In this section all of the contact area is parametrized by cubic B-Spline by using a global description of all the segments. With this technique, the projection of non-mortar points on mortar segments will be described by a unique set of parameters. For that reason, a structured discretization method must be used to reconstruct the mortar surface. In [27], [28] and Piegl L [74] the B-Spline was used initially for the geometrical design and not to improve the mechanical analysis. This sub-chapter includes a methodology which transforms the structured surfaces interpolated with linear finite element in B-Spline curves. We keep a linear Lagrange interpolation for the inside of master body elements. For the border, all the segments will be transformed into a single cubic B-Spline curve (Figure 27).

The discontinuity in the evolution of the normal vector raises problems in the precision of the gap calculation which influence directly the convergence. It presents a supplementary problem to reach a good continuity in the mortar surface which is added to the instabilities in slipping.

Several algorithms in the literature describe the interpolation of a set of points and tangents in a B-Spline curve with a prescribed continuity order (normally higher than $\mathrm{C}^{1}$ ). We pick up the algorithms developed in [27] and [74] and we replace the set of points by all the nodes of the mortar surface (Figure 28).

The mortar surface node $N_{i}^{m}, i=0, \ldots, n$, are going to be interpolated with a cubic B-Spline curve. We attribute to each of these nodes a parameter noted $u_{i} \in[0,1]$. A knots vector, noted $U$ and with a size $m=n+2 p$, contains all the parameters $u_{i}$ of all the nodes to be interpolated. $\mathrm{P}$ is the order of $\mathrm{B}-$ Spline which is equal to 3 for cubic B-Spline. To define $U$ we have:

$$
\begin{aligned}
& u_{0}=\ldots=u_{3}=0, u_{m-3=} \ldots=u_{m=} 1 \\
& u_{k+3}=\frac{1}{3} \sum_{j=k}^{k+2} \bar{u}_{j} \quad k=1, \ldots, n-3
\end{aligned}
$$

With

$$
\bar{u}_{j}=\bar{u}_{j-1}+\frac{\left|N_{j}^{m}-N_{j-1}^{m}\right|}{r} \quad j=1, \ldots, n-1
$$

in which $\mathrm{r}$ is the sum of the length of all mortar segments:

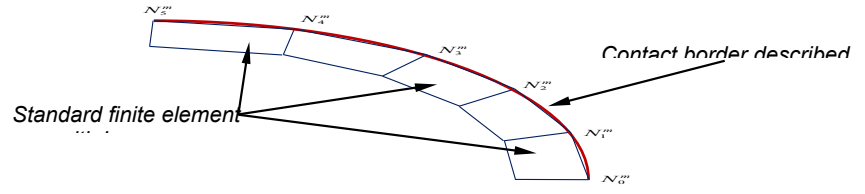

Figure 26: Contact border smoothed with a cubic B-Spline curve.

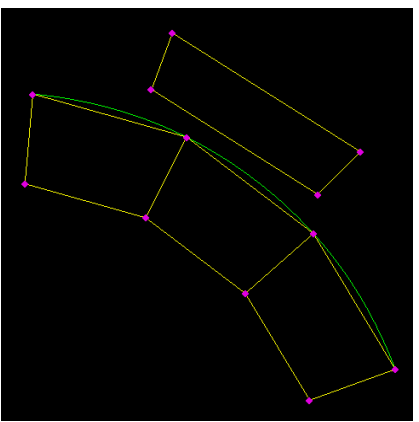

Figure 27: Application of cubic B-Spline in FiEStA code.๑

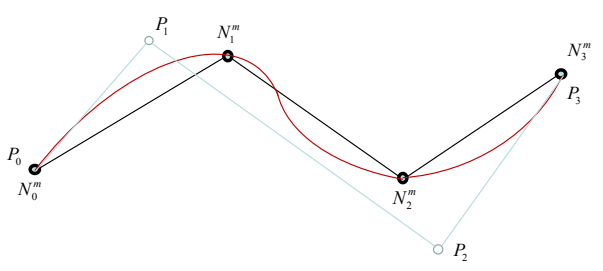

Figure 28: B-spline for an interpolation of 4 nodes.

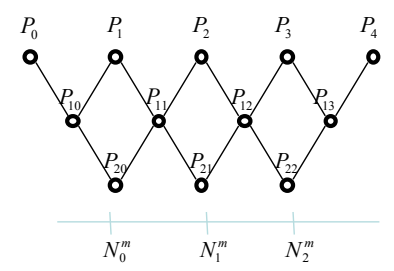

Figure 29: Shape function of a B-Spline.

$r=\sum_{j=1}^{n}\left|N_{j}^{m}-N_{j-1}^{m}\right|$

By using the expressions (3.16) and (3.18), the vector $U$ is given by:

$U=\left\{0,0,0,0, u_{k+3}, \ldots, u_{n}, 1,1,1,1\right\} \quad k=1, \ldots, n-3$

Having defined the knots vector $U$, it remains to calculate control points to build the B-Spline curve (Figure 29) which passes by the nodes $N_{i}^{m}$. We can define a system of equation $(\mathrm{n}+1) \times(\mathrm{n}+1)$ :

$$
N_{i}^{m}=\sum_{i=0}^{n} B_{j}\left(\bar{u}_{i}\right) P_{j}
$$

Under matrix form, we can rewrite (3.20) as follows:

$\left[\begin{array}{ccccc}1 & 0 & \cdots & 0 & 0 \\ B_{0}\left(\bar{u}_{1}\right) & B_{1}\left(\bar{u}_{1}\right) & \cdots & B_{n-1}\left(\bar{u}_{1}\right) & \vdots \\ B_{0}\left(\bar{u}_{2}\right) & B_{1}\left(\bar{u}_{2}\right) & \cdots & B_{n-1}\left(\bar{u}_{2}\right) & \vdots \\ \vdots & \vdots & & \vdots & 0 \\ 0 & B_{1}\left(\bar{u}_{n-1}\right) & \cdots & B_{n-1}\left(\bar{u}_{n-1}\right) & B_{n}\left(\bar{u}_{n-1}\right) \\ 0 & 0 & 0 & 0 & 1\end{array}\right]\left[\begin{array}{c}P_{0} \\ P_{1} \\ \vdots \\ P_{n-1} \\ P_{n}\end{array}\right]=\left[\begin{array}{c}N_{0}^{m} \\ N_{1}^{m} \\ \vdots \\ N_{n-1}^{m} \\ N_{n}^{m}\end{array}\right]$


$P_{j}$ are control points to be calculated and $B_{j, 3}$ the shape functions (From (3.21), control points are obtained with:

$$
\left\{P_{n}\right\}=\left[B_{n}\right]^{-1}\left\{N_{n}^{m}\right\}
$$

Using the expression (3.20) and (3.22), we can write any point of the B-Spline curve as follows:

$$
x^{m}(t)=\sum_{i=0}^{n} B_{i}(t) P_{i}
$$

With $t \in[0,1]$. In particular, if $t$ corresponds to a value in the vector $U$, the calculated point is a mortar node.

We can describe all the points of the B-Spline curve according to a list of control points and a polynomial shape function as shown in the relation (3.23). We detail the mode of calculation of the used shape function and the contents of control points set. In the Figure 30, points $P_{1 i}$ and $P_{2 i}$ are fictitious points used to find a cubic interpolation from a succession of three linear interpolations.

We have to define the expression of a point in the B-Spline curve provided between nodes $N_{0}^{m}$ an $N_{1}^{m}$ d. This expression will be a polynomial of order 3 according to the variable $t$ (Cubic B-Spline).

$$
\begin{aligned}
P= & (1-t) P_{20}+t P_{21} \\
= & (1-t)\left[(1-t) P_{10}+t P_{11}\right]+t\left[(1-t) P_{11}+t P_{12}\right] \\
= & (1-t)\left[(1-t)\left[(1-t) P_{0}+t P_{1}\right]+t\left[(1-t) P_{1}+t P_{2}\right]\right] \\
& +t\left[(1-t)\left[(1-t) P_{1}+t P_{2}\right]+t\left[(1-t) P_{2}+t P_{3}\right]\right]
\end{aligned}
$$

The calculation of the polynomial $P$ depends on control point $P_{\sigma^{\prime}}$ $P_{1}, P_{2}$ and $P_{3}$ and. A modification of the basic geometry for the mortar segment between nodes $N_{1}^{m}$ and $N_{1}^{m}$ does not change the definition of all the B-Spline curve but only the position of four control points which define all the points of the curve on this segment. The expression (3.24) can be rewritten as follows:

$$
P=(1-t)^{3} P_{0}+3 t(1-t)^{2} \mathrm{P}_{1}+3 \mathrm{t}^{2}(1-t) \mathrm{P}_{2}+\mathrm{t}^{3} P_{3}(3.25)
$$

We derive (3.23) to obtain the expression of the tangent vector by applying (3.5):

$$
a^{m}(t)=\sum_{i=0}^{n} B_{i, \xi}(t) P_{i}
$$

To calculate the minimal distance which separates both bodies, it is necessary to verify the projection condition (Figure 31 ):

$$
\overline{\bar{x}^{m}(t) x^{n m}} \cdot a^{m}(t)=0
$$

The normal vector for the B-Spline curve is obtained by applying (3.1). have:

We return to the application of projection condition (3.27), we

$$
\left(x^{n m-} x^{m}\right) \cdot a^{m}=0
$$

To satisfy this condition, we chose to give an expression of $x^{t} x^{m}$ according to the control points which are associated to it and also of the tangent vector $a^{m}$ in this point by using the relation (3.5). This vector can be defined as follows:

$$
a^{m}=-3(1-t)^{2} P_{0}+\left(3-12 t+9 t^{2}\right) P_{1}+\left(6 t-9 t^{2}\right) P_{2}+3 t^{2} P_{3}(3.29)
$$

By applying the relation (3.26) with the relation (3.27) we obtain a polynomial of order 5 to be solved to find the good value of $t$ which verifies the condition of projection. By applying Newton's iterations, we are not going to develop all the terms of (3.26) according to control

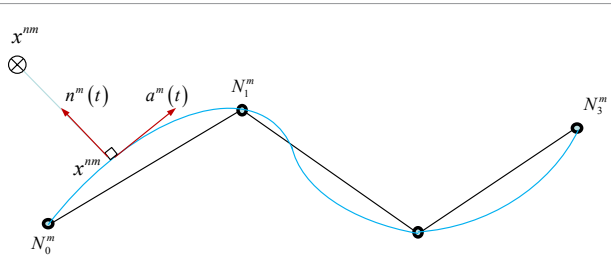

Figure 30: Projection on the mortar surface smoothed by cubic B-Spline.

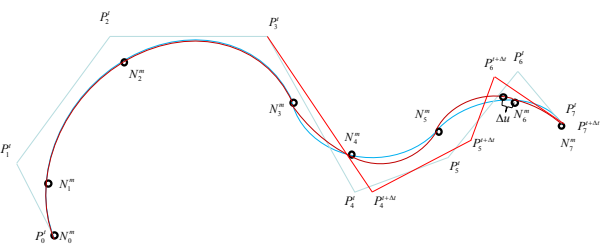

Figure 31: Influence of the change of the control point position.

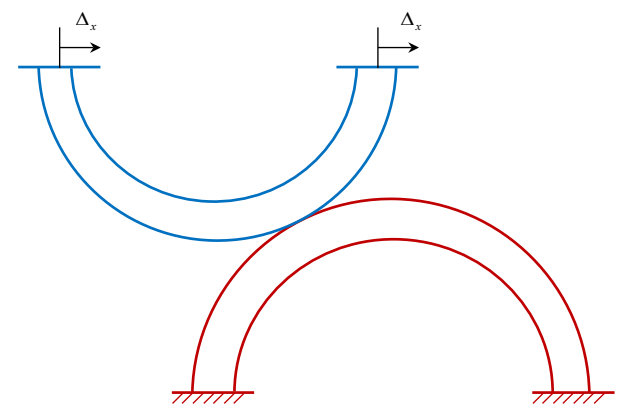

Figure 32: Contact problem between two half-rings.

points to avoid an expression complicated according to the latter. A derivative of the expression (3.26) gives:

$$
-\left(a^{m}\right)^{2}+\left(x^{n m}-x^{m}\right) \cdot a_{, t}^{m}=0
$$

with

$a_{, t}^{m}=(6-6 t) P_{0}+(-12+18 t) P_{1}+(6-18 t) P_{2}+6 t P_{3}$

Now, we have quite the expressions of the terms in (3.30) to calculate the projection by Newton's method.

The application of the $\mathrm{c}$ projection condition in (3.5) gives:

$$
\left(x_{X}^{n m}-x_{X}^{m}\right) a_{X}^{m}-\left(x_{Y}^{n m}-x_{Y}^{m}\right) a_{Y}^{m}=0
$$

By using the expression (3.23) and (3.26), we obtain a polynomial of order 5 according to the variable $t$. Newton's iterations will be essential to determine the good value of $t$ that satisfies the conditions (3.32).

After some iteration, we obtain the value of $t$ which assures the projection. This value is located between two values $u_{i}$ and $u_{i+1}$ in the knots vector $U$. We can calculate the value of the corresponding natural coordinate to define the tangent matrix and the residual vector for the resolution of the contact problem. A variable changing will be necessary in this case, it's applied as follows:

$$
\xi=\frac{t-u_{i}}{u_{i+1}-u_{i}}
$$

During the calculation, mortar nodes can change position. It is necessary to recalculate in this case control points and the knots vector $U$. The modification is then local and not global. A point $x^{m}(u)$ 
on the curve is piloted by four control points in the case of a cubic interpolation. It will not be influenced by a change of a control point position which it does not depend (figure 32).

The smoothing of the mortar surface by cubic B-Spline provides C2 continuity for the entire curve. The displacement field in the element is often defined by a Lagrange interpolation (first order in this study) while the geometry of the border is described by a cubic polynomial. This difference in the degree of interpolation can present an incompatibility between the geometry and the kinematic description.

The calculation of the value of the natural coordinate $\xi$ requires the use of Newton's iterations to satisfy the condition of projection. These iterations have not much influence at the time of search for projection with regard to a not smoothed interface. The addition of these iterations is small with regard to the continuity of the normal and the regularity of the contact pressure which brings this technique.

\section{Example of Application}

We consider two half-rings in contact [70] as shown in the Figure 33. One ring is fixed to its basis when the other one, it moves horizontally with $\Delta_{x} 4 U L$. The same hyper-elastic material described by Néo-Hooke law is considered for both solids. The Young modulus is $E_{\text {ring }}=689.56 \mathrm{~F} / \mathrm{UL}^{2}$ and the Poisson coefficient is $v=0.32$. The inner radius of the fixed ring is $\mathrm{Ri}=10 \mathrm{UL}$ and the outer radius is $\mathrm{Re}=12 \mathrm{UL}$. These dimensions are respectively $\mathrm{Ri}=8 \mathrm{UL}$ and $\mathrm{Re}=10 \mathrm{UL}$ for the other ring.

Materials characteristics :

$$
\begin{aligned}
& E_{\text {ring }}=689.56 \mathrm{~F} / U L^{2} \\
& v=0.32
\end{aligned}
$$

loading

\section{$\Delta_{x}=4 U L$}

We smooth the master surface to solve this problem. Even by using several smoothing techniques, the frictional contact problem remains rather difficult in particular for complex geometry. The role of the smoothing in this case is to move closer the finite elements model to CAD model. This approach can be avoided by using an iso-geometrical model (Figures 34 and 35).

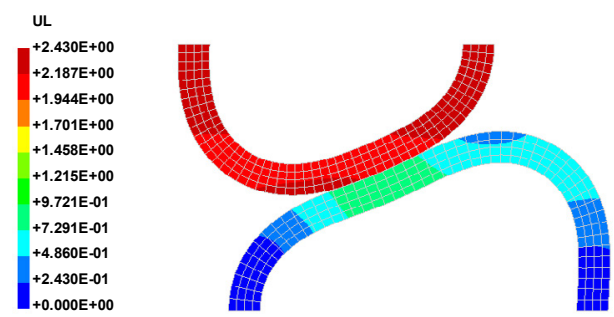

Figure 33: Displacement field for the contact problem between two half-rings.

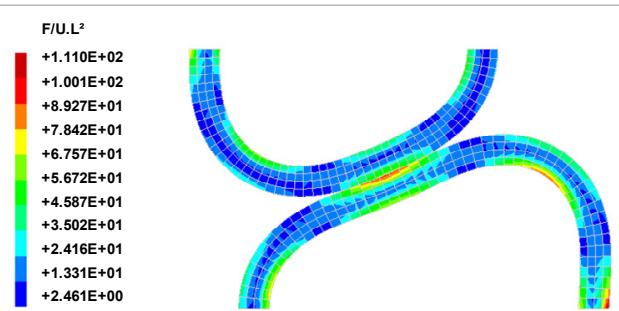

Figure 34: Von Mises stresses for the contact problem between two half-rings.

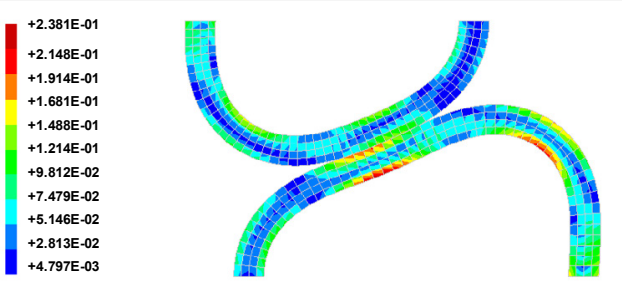

Figure 35: Strain for the contact problem between two half-rings.

All the algorithms detailed in this work are implemented in a house code FiEStA (Finite Elements Structural Analysis). It is a FEM code developed in language $\mathrm{C}++$. The code presents a graphical interface simplified to minimize the time required for the creation of a model.

\section{Conclusion}

This work concerned the simulation and the modeling of a contact problem between deformable two-dimensional solids in finite deformation. We used the mortar method for the discretization of the contact with various formulations for the application of the contact and friction constraints. We examined several numerical examples and in particular contact tests in forming process. We released further to these examples different choices concerning the contact formulation to use and the necessity of discretization improvement in the contact interface. We essentially revisited two points of numerical detail:

A discontinuity of the normal vector evolution in the mortar surface is established and which requires regularization. This need was filled at the beginning by a weighted averaged normal vector in the master surface of the contact. This solution remains restricted to examples of small deformations, that why the use of the Hermit polynomials. An improvement is observed but the contact area does not present a constant continuity that why the use of a cubic B-Spline curve. With this technique, the entire master surface is described by only one surface in which we notice a smooth and continuous evolution of the normal. It also result a better precision in the gap calculation and a remarkable stability of the normal contact pressure.

The use of B-Spline curves for the smoothing of the mortar surface was restricted to the skin of contact and not in all of the body. This choice can generate a kinematic incompatibility between the linear Lagrange discretization inside elements and the cubic description in the interface. A description by B-Spline of the border of all the elements even those who are not in contact will present a consequent loss of calculation time seen that it does not bring additional precision either to the calculation of the gap or to the evolution of the contact effort. A study by using the iso-geometrical analysis to replace the classic meshing and the mortar method for the application of the contact constraints will present a good alternative.

\section{Acknowledgement}

This research was conducted as part of the OASIS project, supported by OSEO within the contract FUI no. F1012003Z.

\section{References}

1. Hallquist JO (1979) Nike2D: An implicit, finite-deformation, finite element code for analysing the static and dynamic response of two-dimensional solids. Technical Report UCRL-52678, University of California, Lawrence Livermore National Laboratory.

2. McDevitt TW, Laursen TA (2000) A mortar-finite element formulation for frictional contact problems. Int J Numerical Method Eng 48:1525-1547.

3. Fischer KA, Wriggers $P(2005)$ Frictionless $2 d$ contact formulations for finite deformations based on the mortar method. Comp Mech 36: 226-244. 
4. Belgacem FB, Hild P, Laborde P (1999) Extensinos of the mortar finite element method to a variational inequality modelling unilateral contact. Mathematical Methods in Applied Sciences 9: 287-303.

5. Wohlmuth BI (2000) A mortar finite element method using dual spaces for the lagrange multiplier. SIAM, J Numerical Analysis 38: 989-1012.

6. Simo JC, Wriggers $P$, Taylor RL (1985) A perturbed Lagrangian formulation for the finite element solution of contact problems. Comp Methods in Appl Mech and Eng 50: 163-180.

7. Hild $P(2000)$ Numerical implementation of two nonconforming finite element methods for unilateral contact. Comp Methods in Appl Mech and Eng184 99-123.

8. Puso MA, Laursen TA (2004) A mortar segment-to-segment contact method for large deformation solid mechanics. Comp Methods in Appl Mech and Eng 193: 601-629.

9. Puso MA, Laursen TA, Solberg J (2008) A segment-to-segment mortar contact method for quadratic elements and large deformations. Comp Methods in Appl Mech and Eng197: 555-566.

10. Hesch C, Betsch P (2009) A mortar method for energy-momentum conserving schemes in frictionless dynamic contact problems. Int $\mathrm{J}$ Numerical Methods in Eng77:1468-1500

11. Tur M, Fuenmayor FJ, Wriggers $P$ (2009) A mortar-based frictional contact formulation for large deformations using Lagrange multipliers. Comp Methods in Appl Mech and Eng 198: 2860-2873.

12. Hüeber S, Wohlmuth BI (2009) Thermo-mechanical contact problems on nonmatching meshes. Comp Methods in Appl Mech and Eng 198: 1338-1350.

13. Puso MA, Laursen TA (2004) A mortar segment-to-segment frictional contact method for large deformations. Computer Methods in Applied Mechanics and Engineering 193: 4891- 4913.

14. Duvaut G, Lions JL (1971) Elasticité avec frottement. Journal de Mécanique 10: $409-420$

15. Duvaut G, Lions JL (1976) Inequalities in Mechanics and Physics. Springer Verlag, Berlin.

16. Curnier A, He QC, Klarbring A (1995) Continuum mechanics modelling of large deformation contact with friction. Contact Mechanics: 145-158.

17. Kikuchi N, Oden JT (1988) Contact problems in elasticity: A study of variationa inequalities and finite element methods. SIAM, Philadelphia.

18. Wriggers $P$, Van $T$ Vu, Stein E (1990) Finite element formulation of large deformation impact-contact problems with friction. Computers \& Structures 37 : 319-331.

19. Wriggers $P$ (2004) The moving friction cone approach for three-dimensional contact simulations. International J Comp Methods 1: 105-119.

20. Heege A, Alart P (1996) A frictional contact element for strongly curved contact problems. Int J Numerical Methods in Eng 39: 165-184.

21. Lengiewicz J, Korelc J, Stupkiewicz1 S (2011) Automation of finite elemen formulations for large deformation contact problems. Int J Numerical Methods in Engineering 85: 1252-1279.

22. Wriggers P, Krstulovic-Opara L, Korelc J (2001) Smooth C1-interpolations for two-dimensional frictional contact problems. Int $\mathrm{J}$ Numerical Methods in Eng 51: 1469-1495

23. Muñoz JJ (2008) Modelling unilateral frictionless contact using the null-space method and cubic B-Spline interpolation. Comput Methods Appl Mech Eng 197: 979-993.

24. Hueber S, Wohlmuth B (2005b) A primal-dual active set strategy for non-linear multibody contact problems. Comp Methods in Appl Mech and Eng194: 31473166.

25. Padmanabhan V, Laursen TA (2001) A framework for development of surface smoothing procedures in large deformation frictional contact analysis. Finite Elements in Analysis and Design 37: 173-198.

26. Kikuchi $\mathrm{N}$ (1982) A smoothing technique for reduced integration penalty method in contact problems. Int J Numerical Methods in Eng 18: 343-350.

27. Farin G (1993) Curves and surfaces for computer aided geometric design. A Practical Guide. Academic Press, Boston.

28. Piegl L, Tiller W (1995) The nurbs Book, Springer, Berlin, Heidelberg.
29. Wriggers P (2006) Computational contact mechanics. (2ndedn), Springer, Verlag.

30. Feng ZQ, Peyraut F, Labed N (2003) Solution of large deformation contact problems with friction between Blatz-Ko hyperelastic bodies. Int J EngSci 41 : 2213-2225.

31. Hueber S, Mair M, Wohlmuth B (2005a),A priori error estimates and an inexact primal dual active set strategy for linear and quadratic finite elements applied to multibody contact problems. Appl Numerical Math 54: 555-576.

32. Hartmann S, Brunssen S, Ramm E, Wohlmuth B (2007) Unilateral nonlinear dynamic contact of thin-walled structures using a primal-dual active set strategy. Int J Numer Meth Eng 70: 883-912.

33. Popp A, Gee MW, Wolfgang A, Wall (2009) A finite deformation $\mathrm{m} \circ \mathrm{r} t \mathrm{a}$ contact formulation using a primal-dual active set strategy. Int J Numer Meth Engng 79: 1354-1391.

34. Hertz H (1882) Uber die Beriihrung fester elastischer Korper. J fur die Reine und Angewandte Mathematik 29: 156-171

35. Johnson C (1987) Numerical solution of partial differential equations by the finite element method. Cambridge University Press.

36. Ciarlet G (1978) The finite element method for elliptic problems. North-Holland Amsterdam.

37. Curnier A, Alart $P$ (1988) A generalized newton method for contact problems with friction. J Mec Theor Appl 7: 67-82

38. Zienkiewicz OC, Taylor RL (1991) The Finite Element Method. (4thedn) McGraw-Hill, London.

39. Francavilla A, Zienkiewicz OC (1975) A note on numerical computation of elastic contact problems. Int J Numerical Methods in Engineering 9: 913-924

40. Hughes TRJ, Taylor RL, Kanoknukulchai W (1977) A finite element method for large displacement contact and impact problems. Formulations and Computational Algorithms in FE Analysis: 468-495.

41. Simo JC, Taylor RL (1985) Consistent tangent operators for rate-independent elastoplasticity. Comp Methods in Applied Mech and Eng 48: 101-118.

42. Simo JC, Laursen TA (1992) An augmented lagrange treatment of contact problems involving friction. Computers and Structures 42: 97-116.

43. Laursen TA, Simo JC (1993b) A continuum-based finite element formulation for the implicit solution of multibody, large deformation frictional contact problems Int J Numerical Methods in Eng 36: 3451-3485.

44. Wriggers P (1994) Finite element algorithms for contact problems. Arch Compt Meth Eng 2: 1-49.

45. Alart P, Curnier A (1991) A mixed formulation for frictional contact problems prone to newton like solution methods. Comp Methods in Appl Mech and Eng 92: 353-375.

46. Taylor RL, Papadopoulos O (1991) On a patch test for contact problems in two dimensions. In: Wriggers P, Wagner W, eds. Nonlinear Comp.

47. El-Abbasi N, Bathe KJ (2001) Stability and patch test performance of contact discretizations and a new solution algorithm. Computers and Structures 79 : 1473-1486.

48. Sauer RA, De Lorenzis L (2013) A computational contact formulation based on surface potentials. Comp Methods in Appl Mech and Eng 253: 369-395.

49. Renaud C, Cros JM, Feng ZQ, FengB (2009) The Yeoh model applied to the modeling of large deformation contact/impact problems. Int J Impact Eng 36 659-666.

50. Crisfield MA (2000) Re-visiting the contact patch test. Int J Numerical Methods in Eng 48: 435-449.

51. Paczelt I, Szabo B, Szabo T (1999) Solution of contact problem using the hp-version of the finite element method. Computers \& Mathematics with Applications 38: 49-69.

52. Fischer KA, Wriggers $P$ (2006) Mortar based frictional contact formulation for higher order interpolations using the moving friction cone. Computer Methods in Applied Mech and Eng.

53. Puso MA (2004) A 3D mortar method for solid mechanics. Int J for Numerical Methods in Eng 59: 315- 336. 
Citation: Kallel A, Bouabdallah S (2017) 2d Frictional B-Spline Smoothed Mortar Contact Problems Part I: Matching Phase. J Appl Mech Eng 6: 272. doi: $10.4172 / 2168-9873.1000272$

54. Yang B, Laursen TA, Meng X (2005) Two dimensional mortar contact methods for large deformation frictional sliding. Int $\mathrm{J}$ Numerical Methods in Eng 62 : 1183-1225.

55. Hueber S, Matei A, Wohlmuth BI (2007) Efficient algorithms for problems with friction. Society for Industrial and Applied Mathematics. Siam J Sci Comput 29: 70-92.

56. Gitterle M, Popp A, Gee MW, Wall WA (2010) Finite deformation frictional mortar contact using a semi-smooth Newton method with consistent linearization. Int $J$ Numerical Methods in Eng 84: 543-571.

57. Bernardi C, Debit N, Maday Y (1990) Coupling finite element and spectral methods: First results. Mathematics of Computation 54: 21-39.

58. Bernardi C, Maday Y, Patera A (1994) A new nonconforming approach to domain decomposition: the motar element method. Nonlinear Partial Differential Equations and their Application. Pitman.

59. Belgacem FB, Hild P, Laborde P (1997) Approximation of the unilateral contact problem by the mortar finite element method. C.R Acad Sci Paris Ser I 324 123-127.

60. Krause $\mathrm{RH}$, Wohlmuth $\mathrm{BI}$ (2002) A dirichlet-neumann type algorithm for contact problems with friction. CVS 5: 139-148.

61. Flemiscli B, Puso MA, Wolilmutli B (2005) A new dual mortar method for curved interfaces: 2d elasticity. Int J Numerical Methods in Eng 63:813-832.

62. Popp A, Gitterle M, Gee MW, Wall WA (2010) A dual mortar approach for 3D finite deformation contact with consistent linearization. Int J Numerical Methods in Eng 83: 1428-1465.

63. Babuska I (1973) The finite element methode with larangian multipliers. Numer Math 20: 179-192.
64. Brezzi $F$ (1974) On the existence, uniqueness ad approximation of saddle-pont problems arising from lagrangian multipliers. RAIRO, Anal Numér 8(R2):129-151.

65. Brezzi F, Fortin M (1991) Mixed and hybrid finite element methods, Springer Verlag, New York.

66. Wohlmuth B, Krause R (2004) Monotone methods on non-matching grids for nonlinear contact problems. SISC 25: 324-347.

67. Wriggers P, Miehe C (1992) On the treatment of contact contraints within coupled thermomechanical analysis. Finite Inelastic Deformations. Springer Berhn.

68. Hueber S, Wohlmuth BI (2009) Thermo-mechanical contact problems on nonmatching meshes. Comp Methods in Appl Mech and Eng 198:1338-1350.

69. Opara LK, Wriggers $\mathrm{P}(2005)$ A three-dimensional contact element based on the moving friction cone approach and the elliptical Coulomb law. VIII International Conference on Computational Plasticity. CIMNE, Barcelona.

70. Foley J, vanDam A, Feiner S, Hughes J (1990) Computer graphics principles and practice. (2ndedn) Addison Wesley Publishing Company.

71. Pietrzak G, Curnier A (1999) Large deformation frictional contact mechanics: Continuum formulation and augmented Lagrangean treatment. Methods in Appl Mech and Eng 177: 351-381.

72. Al-Dojayli M, Meguid SA (2002) Accurate modeling of contact using cubic splines. Finite Elements in Analysis and Design 38: 337-352.

73. Tur M, Giner E, Fuenmayor FJ, Wriggers P (2012) 2D contact smooth formultion based on the mortar method. Comp Methods in Appl Mech and Eng 247:1-14.

74. Piegl L, Tiller W (1997) The Nurbs Book. (2ndedn) Monographs in visual Communication, Springer, Verlag. 